\title{
The histopathological changes at skin of German shepherd dogs associated with ringworm infection in directorate of $\mathrm{K} 9$ in Al-Diwanyia province.
}

\author{
H.Kh.Ul.Al-Karagoly, \\ Coll. of Vet. Med./ Univ. of AL-Qadissiya \\ email: hassank_ulaiwi@yahoo.com
}

\begin{abstract}
The current study was conducted to exam the histopathological changes of skin of German shepherd dogs suffering from ringworm infection. It may be the first study on this breed of dog in the Al-Diwanyia province. Seven German shepherd dogs from directorate of K9 in Al-Diwanyia province suffering from skin lesion were used in this study. Highly contaminated fur of infected dogs was cultured on Sabouraud's dextrose agar (SDA). A five $\mathrm{mm}$ shaved biopsies of skin from all cases were taken from lesions present on shoulder region and after routine processes histological technique to evaluate the histopathological changes. The result were revealed that the colonies of Microsporum canis was flat, spreading, white to faint creamy-coloured with a dense buffy, granular to coarsely fluffy to hairy surface with radial grooves, and the histopathological exam of skin were showed presence of hyperkeratosis, spongiosis and marked dermal edema, skin bullae in the epidermal layer and accumulation of collagen fibers found of spores and hyphae of Microsporum in the stratum basale layer of epidermis and infiltration of eosinophils in the dermis layer directly beneath the basal layer of epidermis.

Key words:skin histopathological alterations, ringworm infection, German shepherd dog.

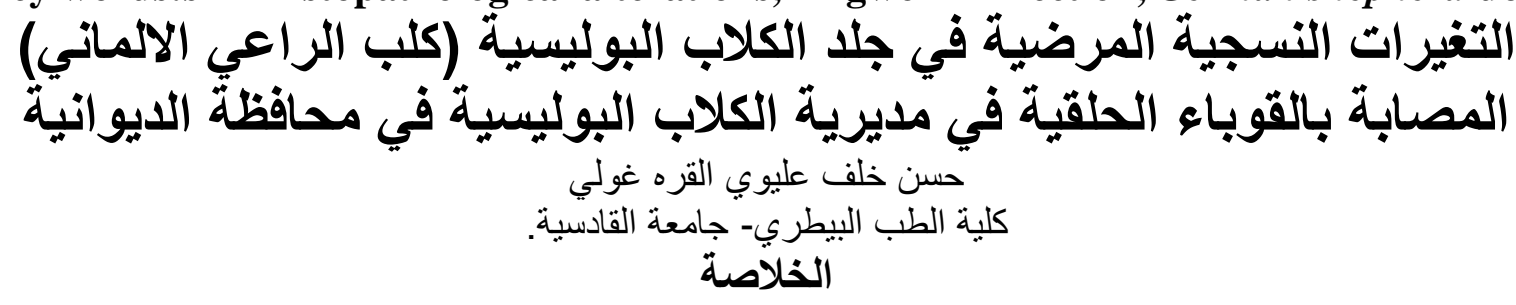

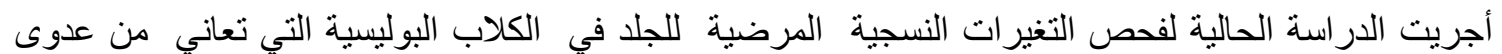

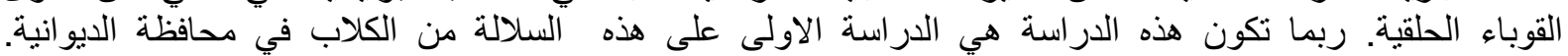

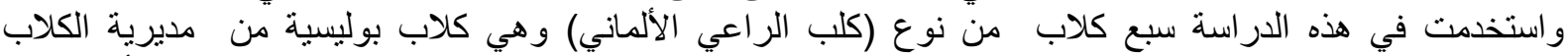

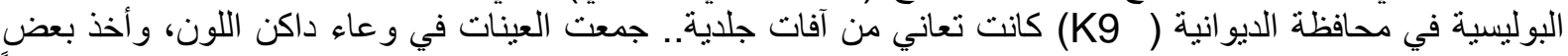

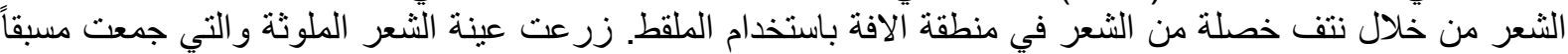

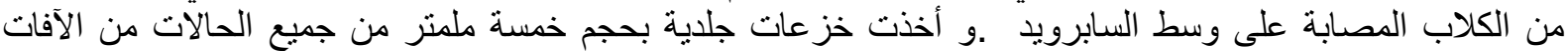

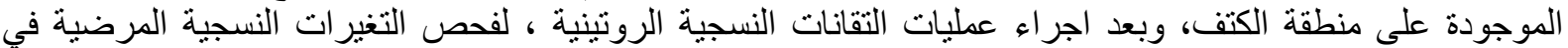

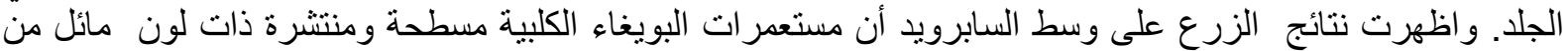

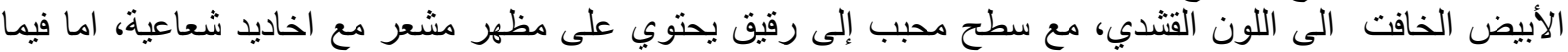

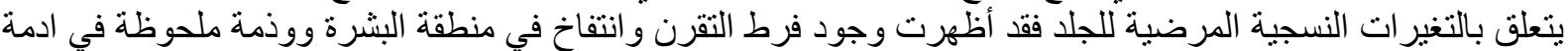

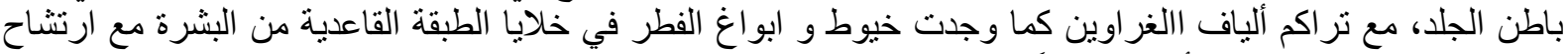

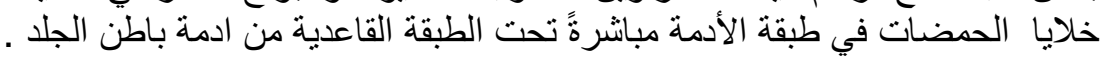

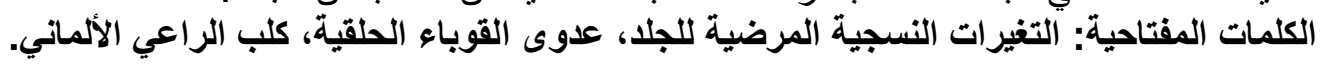

\section{Introduction}

A police dog, in some areas referred to as a "K-9", is a dog that is specifically trained to assist police and other lawenforcement personnel in their work. In this context, German shepherd was the most commonly used breed ${ }^{(1)}$. A very large group of keratinophylic fungi are common inhabitants of the soil and shed from the hairs and skin cells of animals, as well as fall from animals and humans during the natural and continuous cycle of skin and coat 
shedding, but only three genera, known as dermatophytes, are known to cause disease ("ringworm") in animals and humans, that include Microsporum, Trichophyton and Epidermophyton; the first two are most frequently found in animals while the third causes problems mainly in humans ${ }^{(2)}$. These three genera are very important because their particular ability to transmissible to animals and humans and cause a worldwide health problem ${ }^{(3)}$. Dogs and cats can infected with dermatophyte at any age, but most frequent in the young aged animals. poor nutrition, crowding of animals , poor management and insufficiency or an adequate quarantine period for infected pets are most important risk factor in addition to age (4,5). Microsporum canis, followed by M. gypseum and Trichophyton mentagrophytes are the most common fungus isolated from fur of dog and cat. Those three genera are the most reported dermatophytes found worldwide and so-called zoophylic strains ${ }^{(6)}$.

\section{Materials and methods}

Scraping: Seven German shepherd dogs from directorate of $\mathrm{K} 9$ in Al-Diwanyia province suffering from skin lesions were used in this study. The skin scraping samples were taken from the edge of the lesion with a surgical blade. Scrapings were taken very superficially to avoid bleeding. Samples were collected on a container with dark background and some hairs were taken by plucking them off with forceps ${ }^{(6)}$.

\section{1-Culturing:}

A highly contaminated fur of infected dogs was cultured on Sabouraud's dextrose agar (SDA) (Himedia com. -India), that include $4 \%$ glucose, $1 \%$ peptone, $2 \%$ agar together with antibacterial agents a combination of penicillin, streptomycin and cycloheximide with an aerobic culture and incubation. The growth was occurring in about 4 to 7 days at $25^{\circ} \mathrm{C}^{(7)}$.

\section{2-Skin biopsies:}

Skin biopsies were taken by method of Shave biopsies which are quick and do not require sutures for closure. A parallel held blade as used to shave a lesion from the skin surface $^{(8)}$. $0.5 \mathrm{~mm}$ shaved biopsies of skin from all cases were taken from lesion present on shoulder region and fixed in $10 \%$ buffered formalin and sent for histopathological examination by using light microscopy to investigate the morphological alterations ${ }^{(9,10)}$.

\section{Results and discussion}

A flat, spreading, white to faint creamy-coloured colonies of Microsporum canis with a dense buffy, granular to coarsely fluffy to hairy surface with radial grooves. Bright golden yellow to brownish yellow colonies usually occur, but non-pigmented strains also appear. The results of histopathological study showed hyperkeratosis, spongiosis and marked dermal edema (figure: 1), some cases revealed criteria of chronic infection with Microsporum canis by formation of skin bullae in the epidermal layer and accumulation of collagen fibers (figures: 2 and 3) with diapedesis of spores and hyphae of Microsporum into the stratum basale layer of epidermis (figure4) and infiltration of eosinophils in the dermis layer directly beneath the basal layer of epidermis (figure 4). The most frequent mycotic disease in carnivores was Microsporia ${ }^{(11)}$, and in dog and cats the M.canis is the most common species, especially in cats ${ }^{(12)}$. Ringworm lesion occurs on the trunk, extremities and face, and characterized by single or multiple scaly annular lesions with a slightly elevated, scaly and or erythematous edge, sharp margin and central clearing, also, the edges of the lesion contain follicular papules, pustules or vesicles ${ }^{(13)}$. On SDA the characteristics of Microsporum canis colonies were indicated by many researchers that describe a flat, white, fluffy, spreading colony develops within 7 to 14 days, and the characteristic of deep yellow pigment may be observed on the reverse side of a colony on Sabouraud dextrose agar or Dermatophyte Test Media (DTM) ${ }^{(14)}$. Our histopathological results were in accordance with those indicate that histologic sections stained with hematoxylin-eosin showed a discrete neutrophilic infiltrate and dermal edema $24 \mathrm{~h}$ after $M$. canis inoculation into the skin of 
guinea pigs, while five days after inoculation, skin fragments showed acanthosis, hyperkeratosis, spongiosis and marked dermal edema and even in sections stained with hematoxylin-eosin fungal spores and hyphae were observed in the stratum

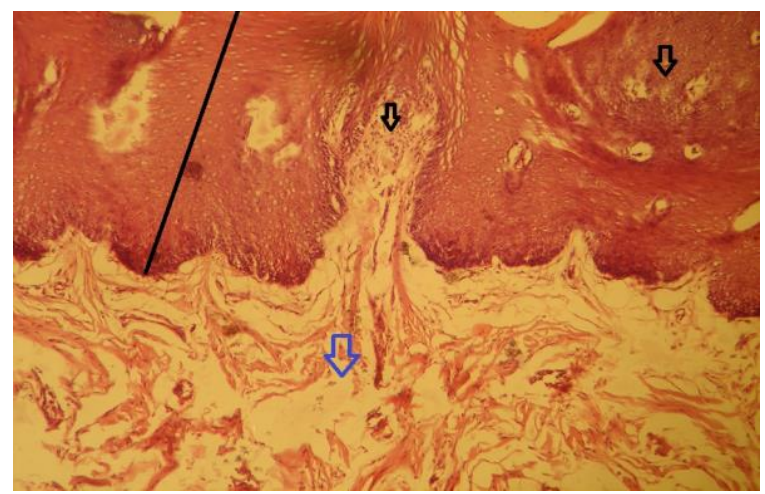

Figure (1): section of skin showed thickening of epidermal layer (black line) with formation of multifocal bullae in the epidermal layer (black arrows), while the dermis layer is edematous (blue arrow). H\&S, 400X.

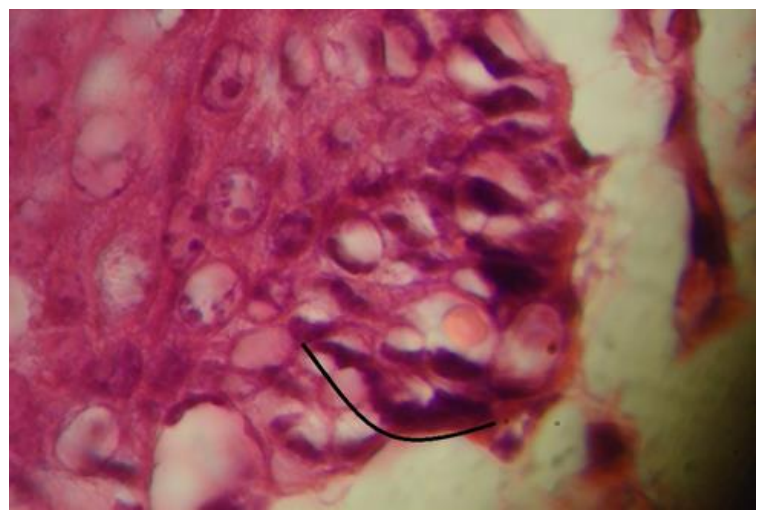

Figure (3): section of skin revealed presence of spores and hyphae (curved black line) between the cells of stratum basale layer of epidermis. H\&E, 1000X.

\section{References}

1-Mesloh, C. (2003). An Examination of Police Canine Use of Force in the State of Florida. A dissertation of Doctor / college of Health and Public Affairs / University of Central Florida/USA.

2-Chretien, J.H. and Garagusi, V.F. (1990). Infections Associated with Pets. Am. Fam. Physician.; 41:831-845 corneum and inside the hair follicles ${ }^{(15)}$, while in our study the spores and hyphae were observed in the stratum basale layer. The formation of skin bullae either due to infection with M.canis ${ }^{(16)}$, or may be due to secondary bacterial infection.

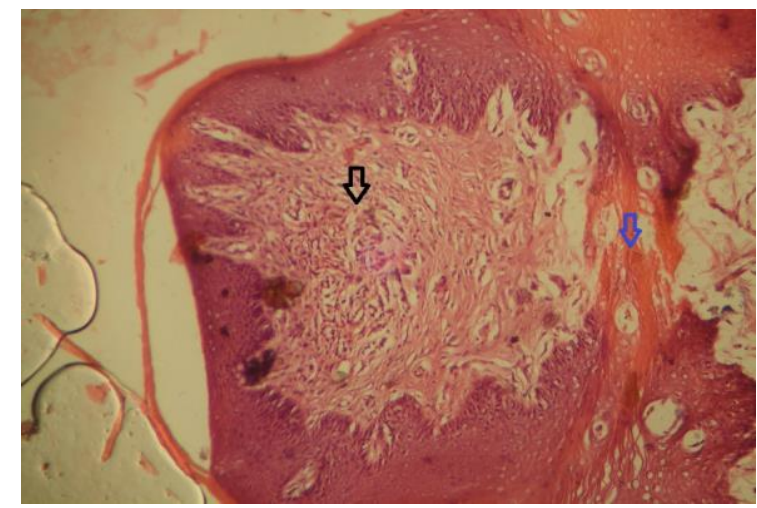

Figure (2): section of skin revealed formation of skin bullae in the epidermal layer of skin (black arrow) with deposition of collagen fibers (blue arrow). H\&S, 400X.

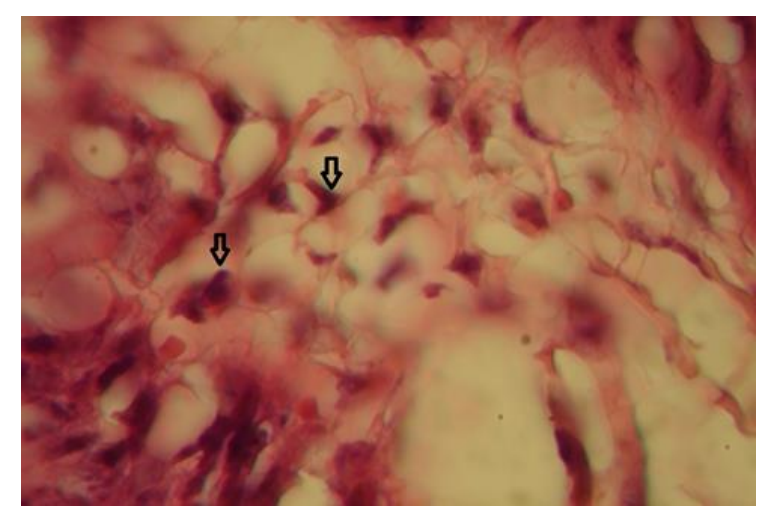

Figure (4): section of skin showed infiltration of eosinophilic cells in dermis layer beneath stratum basale layer. $\mathrm{H} \& \mathrm{E}$, 1000X.

3-Medleau, L. (1991). White-Welther NE. Dermatophytosis in Cats. Compend. Contin. Educ. Prac. Vet.; 13:557-562.

4-Moriello, K.A. and DeBoer, D.J.(1991). Fungal flora of the coat of pet cats. Am J Vet Res.; 52:602-606.

5-Marchisio, V.F.; Gallo, M.G. and Tullio, V. ( 1995). Dermatophytes from cases of skin disease in cats and dogs in Turin, Italy. Mycoses; 38:239-244. 
6-Summerbell, R.C.; Weitzman, I. and Padhye, A.A. (2007). Trichophyton, Microsporum, Epidermophyton, and Agents of Superficial Mycoses. In Murray, P.R. Manual of Clinical Microbiology. (9th Eds.); ASM Press. Washington, DC. Pp: 1874-1897.

7-Hendrikson, D.A. and Krenz, M.M. (1991). Reagents and stains, In: Manual of Clinical Microbiology. (5th Ed.); Washington DC, American Society for Microbiology, USA.Pp:1303.

8-Pflugfeldern, A.; Weide, B.; Eigentler, T.K.; Forschner, A.; Leiter, U.; Held, L.; Meier, F. and Garbe, C. (2010). Incisional biopsy and melanoma prognosis: facts and controversies. Clin Dermatol.; 28(3):316-8.

9-Luna, L.G. (1968). Manual of Histological Staining methods of the Armed Forces Institute of Pathology. (3rd ed.); New York, Mc Graw-Hill.

10-Bancroft, J.D.; Stevens, A. \& Turner, D.R. (1990).Theory and Practice of Histological techniques.(3rd ed.); Churchill Livingstone.PP:21-226.

11-Mederle, N. and Darabus, G.H. (2007). Epidemiological Comparative Inquiry in
Dermatomycosis of Carnivores. LUCRĂRI STIINHIFICE MEDICINĂ VETERINARĂ.; XL: 160-64. \{abstract. \{

12-Hainer, B.L. (2003). Dermatophyte infections. Am Fam Physician; 67:101-8.

13-Cervantes, O.RA. (2003). Ringworm infection in dogs and cats. In: Recent advances in canine infectious diseases. Carmichael L, editor. Ithaca NY: International Veterinary Information Service [IVIS.]

14-Mackenzie, D.W.R. (1963). Hairbrush Diagnosis in detection and eradication of nonflorescent scalp ringworm. Brit Med J.; 2:363365 .

15-Cavalcanti, J.N.; Guerra, J.L.; Gambale, W; Correa, B. and Paula, C. (2002). Histopathologic and mycologic aspects of experimental infection of guinea pigs with Microsporum canis. Braz. J. vet. Res. anim. Sci.; 39(5): 238-243.

16-Aalfs, A. S. and Jonkman, M. F. (2012). Tinea corporis bullosa due to Microsporum canis mimicking linear IgA bullous dermatosis. European Journal of Dermatology; 22(6): 805-6. 